\title{
On Habiro's cyclotomic expansions of the Ohtsuki invariant
}

\author{
Ruth Lawrence and Ofer Ron \\ Einstein Institute of Mathematics, Hebrew University of Jerusalem \\ Givat Ram, 91904 Jerusalem, ISRAEL \\ E-mail address: ruthel@ma.huji.ac.il
}

\begin{abstract}
We give a self-contained treatment of Le and Habiro's approach to the Jones function of a knot and Habiro's cyclotomic form of the Ohtsuki invariant for manifolds obtained by surgery around a knot. On the way we reproduce a state sum formula of Garoufalidis and Le for the colored Jones function of a knot. As a corollary, we obtain bounds on the growth of coefficients in the Ohtsuki series for manifolds obtained by surgery around a knot, which support the slope conjecture of Jacoby and the first author.
\end{abstract}

\section{Introduction}

Suppose that $M$ is a compact oriented 3-manifold without boundary. For any Lie algebra, $g$, and integral level, $k$, there is defined an invariant, $Z_{k+\check{c}_{g}}(M, L)$, of embeddings of links $L$ in $M$, known as the Witten-Reshetikhin-Turaev invariant (see [24], 21]). It is known that for links in $S^{3}, Z_{K}\left(S^{3}, L\right)$ is a polynomial in $q=\exp \frac{2 \pi i}{K}$, namely the generalized Jones polynomial of the link $L$. In this paper we consider only the case $g=s u(2)$.

Now assume that $M$ is a rational homology sphere, with $H=\left|H_{1}(M, \mathbf{Z})\right|$. In the normalization for which the invariant for $S^{3}$ is 1 , denote the invariant for the pair $(M, \emptyset)$, as an algebraic function of $q$ at $K^{\text {th }}$ roots of unity, by $Z_{K}(M)$. For a rational homology sphere $M$ and odd prime $K$, [18] showed that $Z_{K}(M) \in \mathbf{Z}[q]$, so that for some $a_{m, K}(M) \in \mathbf{Z}$, one has

$$
Z_{K}(M)=\sum_{m=0}^{\infty} a_{m, K}(M)(q-1)^{m} .
$$

Although the $a_{m, K}$ are not uniquely determined, it is known from [19] that there exist rational numbers $\lambda_{m}(M)$ such that,

$$
a_{m, K}(M) \equiv \lambda_{m}(M)
$$


as elements of $\mathbf{Z}_{K}$ for all sufficiently large primes $K$. For integer homology spheres, $\lambda_{0}(M)=1$ and $\lambda_{1}(M)=6 \lambda(M)$ where $\lambda(M)$ denotes the CassonWalker invariant of $M$ in Casson's normalization. As a result, one may define a formal power series

$$
Z_{\infty}(M)=\sum_{m=0}^{\infty} \lambda_{m}(q-1)^{m},
$$

with rational coefficients, which is an invariant of rational homology 3spheres, $M$, known as the Ohtsuki series of $M$. This is the asymptotic expansion of the trivial connection contribution to $Z_{K}(M)$ (see [22]). Work of Habiro (see [6], [7]) showed that $Z_{\infty}(M)$ can always be written in the form

$$
Z_{\infty}(M)=\sum_{n=0}^{\infty} a_{n}(q)(q-1)^{n}\{n\} !
$$

where $a_{n}(q) \in \mathbf{Z}\left[q, q^{-1}\right]$. This expression has the advantage that at a root of unity it reduces to a finite sum (polynomial in $q^{ \pm 1}$, while it also can be considered as an element of $\mathbf{Z}[[q-1]]$.

In this paper we review the technique used by Le [14 and Habiro [7, using the $R$-matrix presentation of link and manifold invariants from [20], [21], to compute the colored Jones function for a knot and hence the Ohtsuki series for integer homology spheres obtained by surgery around a knot. We carry out the quantum group calculation in general, obtaining the colored Jones polynomial of a knot as a state sum directly in terms of $q$-numbers as in [3] (though here it is written for arbitrary knot diagrams, not just for braid closures). For arbitrary integer homology spheres the result would be similar, but the knot case is simpler, since then the Gauss diagram involves only one circle.

An outline of the present paper is as follows. In $\S 2$, basic notation is defined relating to $q$-numbers along with a summary of facts needed concerning the quantum group $U_{q} s l_{2}$. In $\S 3$, we follow [21] and Le to give a description of the $s l_{2}$ colored Jones polynomial of a knot, first in terms of $R$-matrices, then using the universal invariant as an algebra element and finally evaluating to get the state sum formula (Theorem 1) for the colored Jones function based on a Gauss diagram description of the knot. In $\S 4$, following Le and Habiro, the transformation from the colored Jones function of a knot to the Ohtsuki series of the 3-manifolds obtained by surgery around the knot, is 
discussed and combining with Theorem 1 gives Habiro's cyclotomic form for the Ohtsuki series. This formulation is used to give bounds on the growth of coefficients in the Ohtsuki series, which support the slope conjecture of [9]. Finally in $\S 5$, the application of the formulae to the trefoil and figure- 8 knots are demonstrated.

\section{$2 q$-numbers and $U_{q} s l_{2}$}

\section{$2.1 \quad q$-numbers}

Let $q=v^{2}=e^{\hbar}$ be a formal parameter. Define $q$-numbers, $q$-factorials and $q$-binomial coefficients according to

$$
[n]=\frac{v^{n}-v^{-n}}{v-v^{-1}}, \quad[n] !=\prod_{i=1}^{n}[i], \quad\left[\begin{array}{c}
n \\
m
\end{array}\right]=\frac{[n] !}{[m] ![n-m] !} .
$$

The dependence on $q$ will be omitted from the notation. We will also define asymmetric $q$-numbers, $q$-factorials and $q$-binomial coefficients according to

$$
\{n\}=\frac{q^{n}-1}{q-1}, \quad\{n\} !=\prod_{i=1}^{n}\{i\}, \quad\left\{\begin{array}{c}
n \\
m
\end{array}\right\}=\frac{\{n\} !}{\{m\} !\{n-m\} !} .
$$

There is an inductive relation as for ordinary binomial coefficients

$$
\left[\begin{array}{l}
n+1 \\
r+1
\end{array}\right]=v^{r-n}\left[\begin{array}{l}
n \\
r
\end{array}\right]+v^{r+1}\left[\begin{array}{c}
n \\
r+1
\end{array}\right], \quad\left\{\begin{array}{l}
n+1 \\
r+1
\end{array}\right\}=\left\{\begin{array}{l}
n \\
r
\end{array}\right\}+q^{r+1}\left\{\begin{array}{c}
n \\
r+1
\end{array}\right\}
$$

from which it follows that the asymmetric $q$-numbers, $q$-factorials and $q$ binomial coefficients all lie in $\mathbf{Z}[q]$. Also, even when $x$ is not an integer, say an element of an algebra, we can make sense of quantum binomial coefficients of the form

$$
\left[\begin{array}{c}
x \\
m
\end{array}\right]=\prod_{i=1}^{m} \frac{[x-i+1]}{[i]}, \quad\left\{\begin{array}{c}
x \\
m
\end{array}\right\}=\prod_{i=1}^{m} \frac{\{x-i+1\}}{\{i\}},
$$

where $m \in \mathbf{N}$. The two sets of $q$-numbers are related by

$$
\{n\}=v^{n-1}[n], \quad\{n\} !=v^{\frac{1}{2} n(n-1)}[n] !, \quad\left\{\begin{array}{c}
n \\
m
\end{array}\right\}=v^{m(n-m)}\left[\begin{array}{c}
n \\
m
\end{array}\right] .
$$

We will also denote by $\{n\}_{-}$, the asymmetric $q$-number obtained by replacing $q$ by $q^{-1}$, and by $\{n\}_{-}$! the corresponding $q$-factorial. Thus $\{n\}_{-}=q^{1-n}\{n\}$ and $\{n\}_{-} !=q^{-\frac{1}{2} n(n-1)}\{n\} !$. 


\subsection{The quantum group $U_{q} s l(2)$}

The quantum group $A=U_{q} s l(2)$ is defined by generators $H, X$ and $Y$ with relations

$$
[X, Y]=[H], \quad[H, X]=2 X, \quad[H, Y]=-2 Y .
$$

Set $K=e^{\hbar H / 4}=v^{H / 2}$. The comultiplication $\Delta: A \longrightarrow A \otimes A$ is given by $\Delta(H)=H \otimes 1+1 \otimes H, \quad \Delta(X)=X \otimes K+K^{-1} \otimes X, \quad \Delta(Y)=Y \otimes K+K^{-1} \otimes Y$, with antipode $S: A \longrightarrow A$ being an antihomomorphism acting on the generators by

$$
S(H)=-H, \quad S(X)=-v X, \quad S(Y)=-v^{-1} Y .
$$

Additionally $A$ is a quasi-triangular Hopf algebra with universal $R$-matrix, $R \in A \otimes A$ satisfying the Yang-Baxter equation $R_{12} R_{13} R_{23}=R_{23} R_{13} R_{12}$. A formula for $R$ (see [2]) is

$R=\sum_{l=0}^{\infty} \frac{\left(1-q^{-1}\right)^{l}}{\{l\} !} q^{\frac{1}{4} H \otimes H} K^{l} X^{l} \otimes K^{-l} Y^{l}=\sum_{l, n=0}^{\infty} \frac{\left(1-q^{-1}\right)^{l}(\hbar / 4)^{n}}{\{l\} ! n !} H^{n} K^{l} X^{l} \otimes H^{n} K^{-l} Y^{l}$

Write this as $R=\sum_{i} \alpha_{i} \otimes \beta_{i}$ (thus the suffix $i$ refers to a pair $(n, l)$ of nonnegative integers). As in any quasi-triangular Hopf algebra, $R^{-1}=(\operatorname{Id} \otimes$ $\left.S^{-1}\right) R$

$$
R^{-1}=\sum_{l, n=0}^{\infty} \frac{(1-q)^{l} q^{\frac{1}{2} l(l-1)}(-\hbar / 4)^{n}}{\{l\} ! n !} H^{n} K^{-l} X^{l} \otimes H^{n} K^{l} Y^{l}
$$

We will write these two formulae together for $\sigma= \pm 1$ as

$$
R^{\sigma}=\sum_{i} \alpha_{i}^{(\sigma)} \otimes \beta_{i}^{(\sigma)}=\sum_{n, l=0}^{\infty} \frac{\left(1-q^{-\sigma}\right)^{l}(\sigma \hbar / 4)^{n}}{\{l\}_{\sigma} ! n !} H^{n} K^{\sigma l} X^{l} \otimes H^{n} K^{-\sigma l} Y^{l}
$$

The square of the antipode is then given by

$$
S^{2}(a)=u a u^{-1}, \quad \text { for all } a \in A,
$$

where $u=m(S \otimes \mathrm{Id}) R_{21}$ and $u S(u)$ is central. Furthermore, $A$ is a ribbon Hopf algebra, that is there is a central (ribbon) element $v$ such that

$$
v^{2}=u S(u), \quad S(v)=v, \quad \epsilon(v)=1, \Delta(v)=\left(R_{12} R_{21}\right)^{-1}(v \otimes v) .
$$


In our case, $K^{2}=v^{-1} u$. This special (charmed) element satisfies the property $S^{2}(a)=K^{2} a K^{-2} \quad \forall a \in A$, while

$$
F \equiv \sum_{i} \alpha_{i} K^{-2} \beta_{i}=\sum_{i} \beta_{i} K^{2} \alpha_{i}=\sum_{l=0}^{\infty} \frac{(q-1)^{l}}{\{l\} !} q^{l^{2} / 2} e^{\hbar H^{2} / 4} K^{-2-2 l} X^{l} Y^{l}
$$

lies in the center of $A$.

\subsection{Finite dimensional $U_{q} s l(2)$-modules}

For each $\mu \in \mathbf{N}$, there is a $\mu$-dimensional module $\Lambda_{\mu}$ with basis $\left\{v_{i}^{(\mu)}\right\}_{i=0}^{\mu-1}$. The quantum group action is described by the lowest weight vector $v_{0}^{(\mu)}$ for which (we omit the superscripts $\lambda$ )

$$
H\left(v_{0}\right)=(1-\mu) v_{0}, \quad Y\left(v_{0}\right)=0, \quad X^{i}\left(v_{0}\right)=v_{i} .
$$

Since $\left[X^{i}, Y\right]=[i][H-i+1] X^{i-1}$, the whole action is given by

$$
H\left(v_{i}\right)=(2 i+1-\mu) v_{i}, \quad X\left(v_{i}\right)=v_{i+1}, \quad Y\left(v_{i}\right)=[i][\mu-i] v_{i-1} .
$$

Since $f \in Z(A)$, it acts as multiplication by a scalar in any irreducible representation. In particular, in $\Lambda_{\mu}, f$ acts by scaling by

$$
f_{\mu}=e^{\hbar(\mu-1)^{2} / 4} v^{\mu-1}=v^{\frac{\mu^{2}-1}{2}},
$$

as can be verified by direct evaluation on $v_{0}$, where $H$ and $K$ act as $1-\mu$ and $v^{\frac{1-\mu}{2}}$ respectively.

\section{The Jones function of a knot}

\subsection{Functorial description}

Suppose $L$ is an oriented framed link in $S^{3}$. The generalized colored Jones polynomial [20] of $L$ is defined when each component $L_{i}$ is colored by a representation $V_{i}$ of $A$ and will be denoted by $J_{L}\left(V_{1}, \ldots, V_{c}\right)$. We will assume for simplicity that $V_{i}$ are irreducible. Indeed, according to [20], there is a functor, $\mathcal{F}$, from the category of (colored) ribbon tangles to the category of vector spaces, under which links (closed tangles) map to scalars, namely $J_{L}$. A slice 

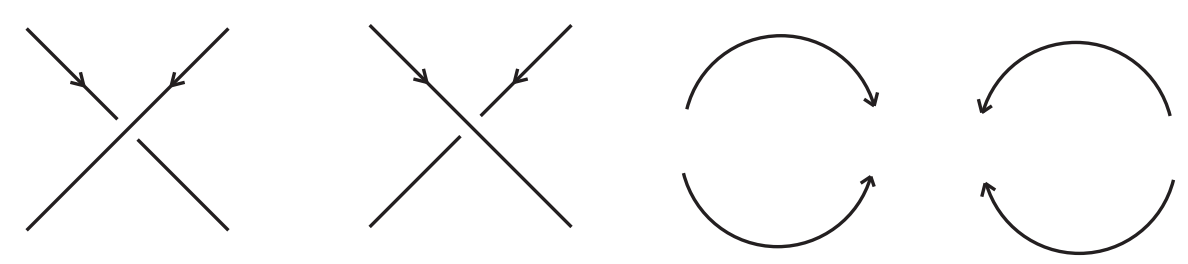

Figure 1: Generators of the category of tangles

of a colored (oriented) ribbon tangle (object in the category) is an ordered list of colors $V_{1}, \ldots, V_{r}$ with orientations $\epsilon_{1}, \ldots, \epsilon_{r}$ and is mapped under the functor $\mathcal{F}$ to $V_{1}^{\epsilon_{1}} \otimes \cdots \otimes V_{r}^{\epsilon_{r}}$, where $V^{-}$denotes the dual representation $V^{*}$. This functor is defined by its images on the generators shown in Figure 1. In our conventions, downward oriented strands are counted positively in objects, while tangle morphisms are composed considering them from bottom to top.

The two orientations of crossing, in which the strands are labeled with representations $V$ and $W$ as shown, have as images the maps $V \otimes W \longrightarrow W \otimes V$ given by $P \circ R$ and $R^{-1} \circ P$, respectively, where $P$ is the permutation of the factors. The four cup and cap sections transform to maps

$$
\begin{array}{rlrl}
V^{*} \otimes V & \longrightarrow \mathbf{C} & V \otimes V^{*} \longrightarrow \mathbf{C} \\
(x, y) & \longmapsto x(y) & (y, x) \longmapsto x\left(K^{2} y\right)
\end{array}
$$

and

$$
\begin{array}{rlrl}
\mathbf{C} & \longrightarrow V \otimes V^{*} & \mathrm{C} \longrightarrow V^{*} \otimes V \\
1 & \longmapsto \sum_{i} e_{i} \otimes e^{i} & & 1 \longmapsto \sum_{i} K^{-2} e^{i} \otimes e_{i}
\end{array}
$$

respectively. Here $\left\{e^{i}\right\}$ is the dual basis for $V^{*}$ to the basis $\left\{e_{i}\right\}$ for $V$.

Suppose that $L$ has one component (knot) and $T$ is a 1-tangle presentation of $L$, that is $T \in \operatorname{Morph}(a, a)$ (where $a$ is the object consisting of one downward oriented point) such that its closure with blackboard framing is ambient isotopic to $L$. Then the above prescription can be used to compute $\mathcal{F}(T)$ (when $a$ and $T$ are colored by $V$ ) as a map $V \longrightarrow V$, for any representation $V$ (the color of the one open strand). This map commutes with 
the action of $A$, so that if $V$ is irreducible it is given by multiplication by a scalar, namely

$$
J_{L}^{\prime}(\mu)=\frac{J_{L}\left(\Lambda_{\mu}\right)}{J_{U}\left(\Lambda_{\mu}\right)}
$$

where $U$ is the unknot with framing zero. For $U$ we have

$$
J_{U}\left(\Lambda_{\mu}\right)=\operatorname{tr}_{\Lambda_{\mu}}\left(K^{2}\right)=\operatorname{tr}_{\Lambda_{\mu}}\left(K^{-2}\right)=v^{\mu-1}+v^{\mu-3}+\cdots+v^{1-\mu}=[\mu] .
$$

By construction, $J_{L}^{\prime}$ is multiplicative under connect sum on $L$ (corresponding to composition of tangles $T$ ). In particular, the algebra element corresponding to a trivial 1-tangle with framing 1 is $f$, so that $J_{L}^{\prime}$ changes by a factor $\left(f_{\mu}\right)^{t}$ under a framing change of $t$ in $L$.

\subsection{Universal $s l(2)$ formulation of Jones polynomial of a knot}

The above functorial prescription for $J_{L}^{\prime}$ when $L$ is a knot expressed as the closure of a 1-tangle $T$, may be rewritten algebraically as follows. First assume that $T$ is presented as a tangle diagram in generic position, in which the crossings are between downward oriented strands (this is always possible). Recall that $R^{\sigma}=\sum_{i} \alpha_{i}^{(\sigma)} \otimes \beta_{i}^{(\sigma)}$ where the sum is over $i \in I$ an indexing set (in this case, pairs of non-negative integers $(n, l)$ ). Place an element of $I$ at each crossing. In the neighborhood of each crossing decorate the crossing stands by elements of $A$ according to Figure $2 ; \alpha_{i}^{\sigma}$ on the overcrossing arc and $\beta_{i}^{\sigma}$ on the undercrossing arc, where $\sigma$ denotes the sign of the crssing ( + for a slash crossing and - for a backslash crossing). At local maxima/minima oriented leftwards decorate the strand by $K^{2}, K^{-2}$ respectively; rightward oriented cups and caps receive no decoration.

Then $\mathcal{F}(T)$ is the evaluation in the chosen representation of the algebra element read off the diagram by tracing the tangle strand according to its orientation, and writing down the decorations in the form of a product from left to right, and then summing over all labels $i$ at crossings. In fact, in the correct quotient of $A$, the algebra element itself is an invariant (this is the universal $U_{h} s l(2)$ invariant); however we do not need this here.

In a general tangle diagram, crossings need not always occur only between downward oriented strands. However, other crossings can always be redrawn in terms of such downward oriented crossings, at the cost of introducing extra caps and cups, by "internally rotating" the crossing. For example a crossing 

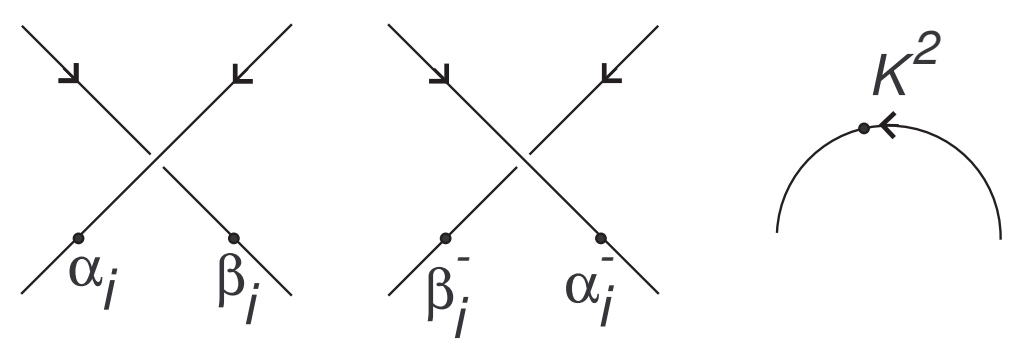

Figure 2: Decorations on 1-tangle elements

of two upward oriented strands can be obtained by "internally rotating" a downward oriented crossing through $\pi$, either clockwise or anticlockwise and consistency of the results follows from the fact that $\left(S^{2} \otimes S^{2}\right) R=R$ along with $S^{2}(a)=K^{2} a K^{-2} \forall a \in A$.

There are altogether eight crossing types given by the orientations on the two strands as well the sign of the crossing itself. The result is that for an arbitrarily oriented crossing, the undercrossing arc is decorated with $\beta_{i}^{(\sigma)}$ while the overcrossing arc is decorated with $\alpha_{i}^{(\sigma)}$ except in the case of a left pointing crossing in which case it is decorated by $S^{2 \sigma}\left(\alpha_{i}^{(\sigma)}\right)$.

\subsection{Gauss diagram formulation of Jones function of a knot}

We will now proceed to carry out the above prescription at the quantum group level for an arbitrary 1-tangle, producing a prescription for $J_{L}^{\prime}(\mu)$ as a state sum of scalar quantities.

The combinatorial data involved in specifying a knot $L$, presented as a 1-tangle closure can be contained in its Gauss diagram. That is, consider the knot as parametrised by an (orieted) circle. Each crossing in $L$ corresponds to exactly two points on the circle, one for the underpass and the other for the overpass. Encode this by joining the points on the circle by an oriented chord from overpass to underpass, marked by a sign to denote the sign of the crossing. The resulting diagram is known as the Gauss diagram of $L$; it is an oriented signed chord diagram on an oriented circle. This is now sufficient 

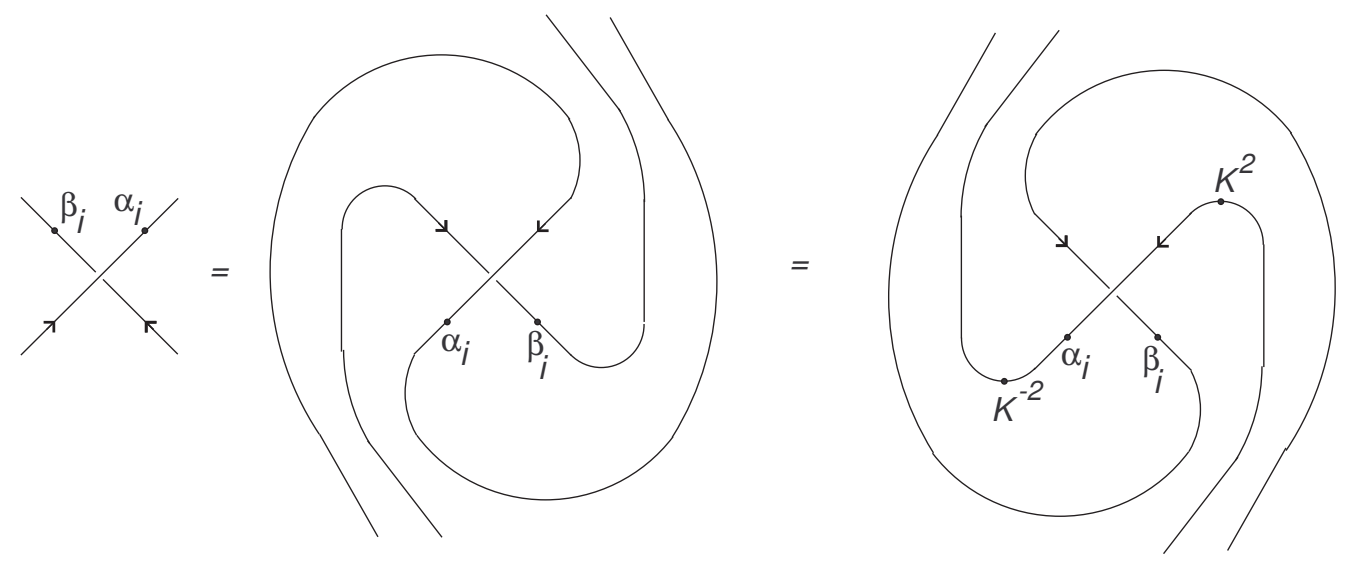

Figure 3: Internal rotation of a crossing
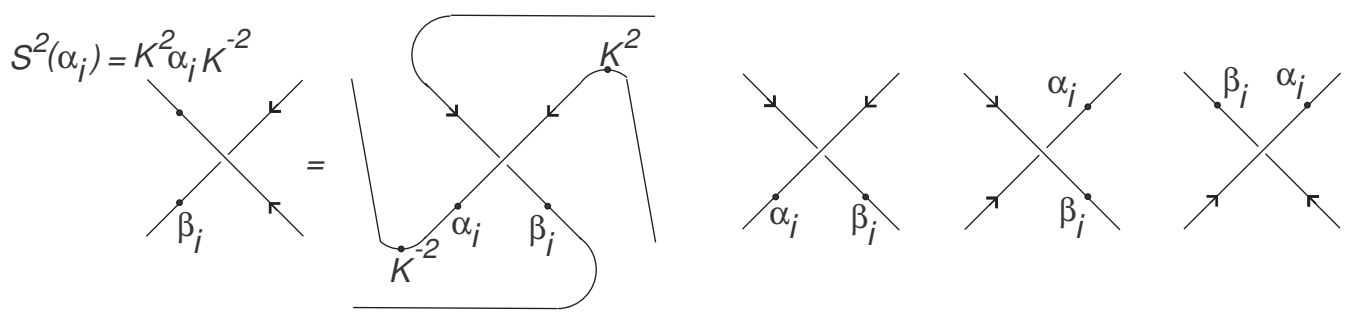

Figure 4: Decorations on all orientations of a positive crossing 


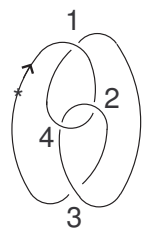

knot

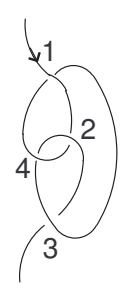

long knot

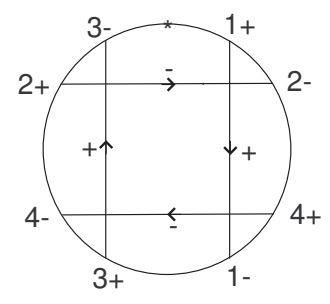

Gauss diagram

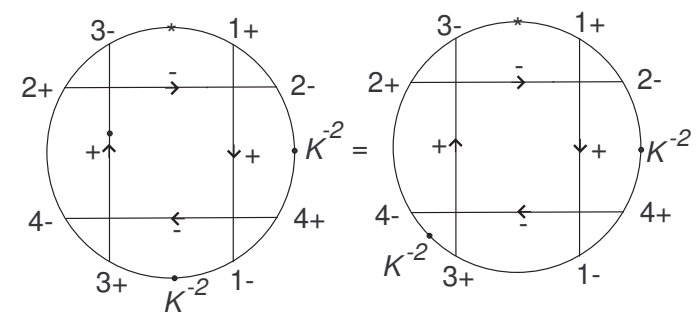

Enhanced Gauss diagram

Figure 5: Gauss diagrams and enhanced Gauss diagrams

data to encode the knot.

Unfortunately the above description of the algorithm for $J_{L}^{\prime}(\mu)$ requires also a tangle presentation with left pointing crossings/cups/caps marked. This requires that the circle be marked with a basepoint as well as blobs $K^{ \pm 2}$ at left pointing cups/caps with additionally left pointing crossings marked. (Note that only left pointing cups/caps/crossings in the long knot are marked; these may differ from those in the closure). Since $S^{2 \sigma}(a)=K^{2 \sigma} a K^{-2 \sigma}$, instead of marking a chord as "left pointing" (whose corresponding crossing was left pointing), one may equivalently place $K^{2 \sigma}$ and $K^{-2 \sigma}$ blobs on the outer circle just before and after the outward pointing (corresponding to the overcrossing arc) end of that chord. The result is an enhanced Gauss diagram in which additional marks have been placed on the outer circle, namely the basepoint and blobs $K^{ \pm 2}$. Figure 5 shows an example for the figure 8 knot.

Let $D$ be such an enhanced Gauss diagram for a knot $K$ with $c$ crossings. In order to write formulae more easily, we give complete combinatorial data for $D$. Number the crossings (chords of $D$ ) by $j=1, \ldots, c$. Let $\sigma_{j}$ denote the sign (orientation) of the crossing, positive for a slash crossing pointing up/down. As we go around the circle, we encounter $2 c$ contributions from crossings which we will index by $i=1, \ldots, 2 c$; for each such $i$ there is a pair $\left(j(i), \epsilon_{i}\right)$ giving the crossing number $j=j(i)$ and the sign with which it is encountered, $\epsilon_{i}$, which is positive for an overcrossing and negative for an undercrossing. Finally let $i_{1}, \ldots, i_{b}$ denote the positions of the blobs $K^{ \pm 2}$, the $a^{\text {th }}$ blob appearing between the $i_{a}^{\text {th }}$ and $\left(i_{a}+1\right)^{\text {th }}$ positions on the circle, with contribution $K^{2 \delta_{a}}$. Throughout the remainder of this paper, we will always use $j$ as an index for crossings and $i, k$ as indices for vertices on the 
Gauss diagram.

The evaluation of $\mathcal{F}(T)$ in the representation $\Lambda_{\lambda}$ may be carried out by computing the action on the lowest weight vector $v_{0}^{(\lambda)}$ (equivalently it is the 00 matrix element), and then

$$
J_{L}^{\prime}(\mu)=\sum_{\substack{n_{j}, l_{j}=0 \\ j=1, \ldots, c}}^{\infty}\left(\prod_{j=1}^{c} \frac{\left(1-q^{-\sigma_{j}}\right)^{l_{j}}\left(\sigma_{j} \hbar / 4\right)^{n_{j}}}{\left\{l_{j}\right\}_{\sigma_{j}} ! n_{j} !}\right) W_{D}^{\mu}(\mathbf{n}, \mathbf{l}) .
$$

Here $W_{D}^{\mu}(\mathbf{n}, \mathbf{l})$ is the 00 matrix element in the evaluation on $\Lambda_{\mu}$ of the quantum group word $W_{D}(\mathbf{n}, \mathbf{l})$ obtained by tracing round the outer circle of $D$ starting at the basepoint, with contributions of $H^{n_{j}} K^{\sigma_{j} l_{j}} X^{l_{j}}$ (outward arrow/overcrossing), $H^{n_{j}} K^{-\sigma_{j} l_{j}} Y^{l_{j}}$ (inward arrow/undercrossing) and $K^{ \pm 2}$ (blobs on the circle), written in order from left to right. It is convenient to use the notation $X^{+}=X$ and $X^{-}=Y$. Using

$$
\left(X^{\epsilon}\right)^{l} H=(H-2 \epsilon l)\left(X^{\epsilon}\right)^{l}, \quad\left(X^{\epsilon}\right)^{l} K=v^{-\epsilon l} K\left(X^{\epsilon}\right)^{l},
$$

we push all powers of $X$ and $Y$ to the end of the word, obtaining $W_{D}(\mathbf{n}, \mathbf{l})=$ $A(\mathbf{n}, \mathbf{l}) B(\mathbf{l})$ where

$$
B(\mathbf{l})=\prod_{i=1}^{2 l}\left(X^{\epsilon_{i}}\right)^{l_{j(i)}}
$$

the product being written from left to right. (Note that $B(\mathbf{l})$ is independent of $\left.n_{1}, \ldots, n_{c}.\right)$ The term $A(\mathbf{n}, \mathbf{l})$ is the product of $H^{\prime}$ 's and $K^{\prime} s$ remaining after all the powers of $X$ and $Y$ have been pushed to the right, namely

$A(\mathbf{n}, \mathbf{l})=\left[\prod_{i=1}^{2 c}\left(H-2 \sum_{k<i} \epsilon_{k} l_{k}\right)^{n_{i}}\left(\prod_{k<i} v^{-\epsilon_{k} l_{k} \cdot \epsilon_{i} \sigma_{i} l_{i}}\right) K^{\epsilon_{i} \sigma_{i} l_{i}}\right] \prod_{a=1}^{b}\left(\prod_{k<i_{a}} v^{-\epsilon_{k} l_{k} \cdot 2 \delta_{a}}\right) K^{2 \delta_{a}}$.

where by abuse of notation we have written $l_{i}$ and $n_{i}$ in place of $l_{j(i)}$ and $n_{j(i)}$ respectively. Collecting terms and noting that $\sum_{i} \epsilon_{i} \sigma_{i} l_{i}=0$,

$$
A(\mathbf{n}, \mathbf{l})=v^{-\sum_{i} \sum_{k<i} \epsilon_{i} \epsilon_{k} \sigma_{i} l_{i} l_{k}-2 \sum_{a} \sum_{k<i_{a}} \delta_{a} \epsilon_{k} l_{k}} K^{2 \sum_{a} \delta_{a}} \prod_{i=1}^{2 c}\left(H-2 \sum_{k<i} \epsilon_{k} l_{k}\right)^{n_{i}} .
$$

Since both $A(\mathbf{n}, \mathbf{l})$ and $B(\mathbf{l})$ preserve the weight, $W_{D}^{\mu}(\mathbf{n}, \mathbf{l})=A^{\mu}(\mathbf{n}, \mathbf{l}) B^{\mu}(\mathbf{l})$ where the superscripts $\mu$ denote the 00 matrix element in the evaluation on 
$\Lambda_{\mu}$. Thus $A^{\mu}(\mathbf{n}, \mathbf{l})$ is obtained from the above expression by replacing all occurrences of $H$ and $K$ by $1-\mu$ and $v^{\frac{1-\mu}{2}}$, respectively. We may now carry out the sum over $n_{1}, \ldots, n_{c}$ in (11) for $J_{L}^{\prime}(\mu)$, since the sum on $n_{j}$ may be taken out to give a factor (where $\lambda=\mu-1$ )

$$
\left[\sum_{n_{j}=0}^{\infty} \frac{\left(\sigma_{j} \hbar / 4\right)^{n_{j}}}{n_{j} !}\left(H-2 \sum_{k<j+} \epsilon_{k} l_{k}\right)^{n_{j}}\left(H-2 \sum_{k<j-} \epsilon_{k} l_{k}\right)^{n_{j}}\right]_{00}=v^{\frac{\sigma_{j}}{2}\left(\lambda+2 \sum_{k<j+} \epsilon_{k} l_{k}\right)\left(\lambda+2 \sum_{k<j-} \epsilon_{k} l_{k}\right)}
$$

Moving to the zero framed knot introduces a framing correction of $f_{\mu}^{-\sum_{j} \sigma_{j}}$, that is $\prod_{j} v^{-\frac{\sigma_{j}}{2}\left(\mu^{2}-1\right)}$. The contribution of the term $A^{\mu}(\mathbf{n}, \mathbf{l})$ to $J_{L_{0}}^{\prime}(\mu)$ becomes simply a power of $v$. Substituting into (II), we get the zero framed colored Jones polynomial as

$$
J_{L_{0}}^{\prime}(\mu)=\sum_{\substack{l_{j}=0 \\ j=1, \ldots, c}}^{\infty}\left(\prod_{j=1}^{c} \frac{\left(1-q^{-\sigma_{j}}\right)^{l_{j}}}{\left\{l_{j}\right\}_{\sigma_{j}} !}\right) v^{a^{\mu}(\mathbf{l})} B^{\mu}(\mathbf{l}),
$$

where (again $\lambda=\mu-1)$

$$
\begin{aligned}
a^{\mu}(\mathbf{l})= & -\sum_{i} \sum_{k<i} \epsilon_{i} \epsilon_{k} \sigma_{i} l_{i} l_{k}-2 \sum_{a} \sum_{k<i_{a}} \delta_{a} \epsilon_{k} l_{k}-\lambda \sum_{a} \delta_{a} \\
& +\sum_{j} \sigma_{j}\left(-\lambda+\lambda \sum_{k<j-} \epsilon_{k} l_{k}+\lambda \sum_{k<j+} \epsilon_{k} l_{k}+2\left(\sum_{k<j-} \epsilon_{k} l_{k}\right)\left(\sum_{k<j+} \epsilon_{k} l_{k}\right)\right)
\end{aligned}
$$

The function $a^{\mu}(\mathbf{l})$ is a linear function of $\mu$, and depends quadratically on the parameters $l_{j}$. Looking at only the parity of terms (that is, mapping onto $\mathbf{Z} / 2 \mathbf{Z}[\mu])$

$$
a^{\mu}(\mathbf{l}) \equiv \sum_{i} \sum_{k<i} \epsilon_{i} \epsilon_{k} l_{i} l_{k}+\left(b+c+\sum_{i} \sum_{k<i} l_{k}\right)(\mu-1)
$$

Since $\sum_{i} \epsilon_{i} l_{i}=0$ (each term $l_{j}$ appears twice with opposite signs), the first term is $-\sum_{j} l_{j}^{2}$. Reversing the order of summation in the last term, $\sum_{i} \sum_{k<i} l_{k} \equiv \sum_{i} i l_{i} \equiv \sum_{j} p_{j} l_{j}$, where $p_{j}$ is the number of segments on the Gauss diagram between the two occurrences of the crossing $j$, that is between $j+$ and $j-$. Observing that $b+c$ is always even and that $p_{j}$ is always odd (as follow from changing the signs of crossings in the knot diagram so as 
to obtain an unknot and then applying Reidemeister moves to untie it and observing that in the process these parities are preserved), it follows that

$$
a^{\mu}(\mathbf{l}) \equiv \mu \sum_{j} l_{j} \quad(\bmod 2) .
$$

The final evaluation required in order to remove all traces of the quantum group $A$ from our formula for $J_{L_{0}}^{\prime}(\mu)$ is that of $B^{\mu}(\mathbf{l})$ in (2), namely the 00 matrix element in the representation $\Lambda_{\mu}$ of the product of terms $X^{l_{i}}$ and $Y^{l_{i}}$ described by the Gauss diagram. Using the explicit form for the basis for $\Lambda_{\mu}$, one can read the product $B(\mathbf{l})$ from right to left, each term $X$ pushes up the level, while each $Y$ pushes it down with a factor $[i][\mu-i]$ starting at level $i$. Reading instead from left to right, $X$ decreases the level while $Y$ raises the level and introduces the factor $[i+1][\mu-1-i]$ when starting from level $i$. Thus we obtain

$$
B^{\mu}(\mathbf{l})=\prod_{j=1}^{c} \prod_{i=s(j)}^{s(j)+l_{j}-1}[i+1][\mu-1-i]
$$

where $s(j)=-\sum_{i<j-} l_{i} \epsilon_{i}$ is the level at the start of the term $Y^{l_{j}}$ contributed by $j-$ in the Gauss diagram (if any $s(j)$ is negative, the expression vanishes). Writing $[i+1][\mu-1-i]=v^{-\sigma \mu}\{i+1\}_{\sigma}\{\mu-1-i\}_{\sigma}$, we obtain

$$
B^{\mu}(\mathbf{l})=\prod_{j=1}^{c} v^{-\mu l_{j} \sigma_{j}} \frac{\left\{s(j)+l_{j}\right\}_{\sigma_{j}} !}{\{s(j)\}_{\sigma_{j}} !}\{\mu-s(j)-1\}_{\sigma_{j}} \cdots\left\{\mu-s(j)-l_{j}\right\}_{\sigma_{j}} .
$$

Substituting into (3) and putting $c^{\mu}(\mathbf{l})=\frac{1}{2} a^{\mu}(\mathbf{l})-\frac{1}{2} \mu \sum_{j} \sigma_{j} l_{j} \in \mathbf{Z}\left[\mu, l_{j}\right]$, we obtain the following result.

Theorem 1 [3] The colored Jones function of a zero framed knot is given by

$J_{L_{0}}^{\prime}(\mu)=\sum_{\substack{l_{j}=0 \\ j=1, \ldots, c}}^{\infty} q^{c^{\mu}(\mathbf{l})} \prod_{j=1}^{c}\left\{\begin{array}{c}s(j)+l_{j} \\ l_{j}\end{array}\right\}_{\sigma_{j}}\left(q^{\sigma_{j}(\mu-s(j)-1)}-1\right) \cdots\left(q^{\sigma_{j}\left(\mu-s(j)-l_{j}\right)}-1\right)$, where $s(j)=-\sum_{i<j-} \epsilon_{i} l_{j(i)}$ and $c^{\mu}(\mathbf{l})$ is a function dependent on the Gauss diagram, linear in $\mu$ and quadratic in $l_{j}$, with integer coefficients, given explicitly by (4) and (5) below.

Several remarks and standard facts about the colored Jones function follow immediately from this theorem. 
- In [3, this theorem is only stated explicitly for braid closures. A very similar (though inequivalent) state sum for the colored Jones function was recently obtained in Theorem 7, [4].

- Considering $q=1+h$, each term can be thought of as a power series in $h$ whose coefficients are polynomials in $\mu$. The contribution from a particular set of $c$ non-negative integers $l_{j}$ is divisible by $h^{\sum_{j} l_{j}}$. That is, the coefficient of $h^{N}$ comes only from $l_{j}$ 's with $\sum_{j} l_{j} \leq N$, a finite number of contributions. So $J_{L_{0}}^{\prime}(\mu)$ is meaningful as a formal power series in $h$ whose coefficients are polynomials in $\mu$ (even when $\mu$ is not an integer); this is the Jones function.

- If $\mu \in \mathbf{N}$, then the contribution from any term for which $s(j) \geq \mu-1$ will vanish. Thus only a finite number of l's will contribute, giving a polynomial; this is the colored Jones polynomial.

- For each $l$, the contribution is a polynomial in $q$ and $q^{\lambda}$, so that $J_{L_{0}}^{\prime}(\lambda)$ can be written as a power series in $h$ whose coefficients are polynomials in $q^{\lambda}$. (See the Melvin-Morton-Rozansky conjecture of [17, 23] proved in [1].)

- Since $\left\{\begin{array}{c}n \\ m\end{array}\right\} \in \mathbf{Z}[q]$, it follows that the contribution from $l$ is divisible by $h^{\sum_{j} l_{j}} \prod_{j}\{j\}$ !. At a root of unity of order $K$, only terms with $l_{j}<K$ $\forall j$ will contribute. Again there are a finite number of contributions.

- The coefficient of $\mu$ in $c^{\mu}(\mathbf{l})$ simplifies to

$$
d(\mathbf{l})=\sum_{j} q_{j} l_{j}-\frac{1}{2} \sum_{a} \delta_{a}-\frac{1}{2} \sum_{j} \sigma_{j}
$$

where $q_{j}=\frac{1}{2} \sum_{i>j+} \sigma_{i}-\frac{1}{2} \sum_{i>j-} \sigma_{i}-\frac{1}{2} \sigma_{j} \in \mathbf{Z}$. When the knot is presented in a blackboard framing which is also zero framed, then $\sum_{j} \sigma_{j}=0$, so that

$$
d(\mathbf{l})=\sum_{j} q_{j} l_{j}-\frac{1}{2} \sum_{a} \delta_{a}
$$

where now $q_{j}$ has the meaning of half the sum of the signs of crossings encountered when tracing around the knot from the overcrossing arc of 
the $j^{\text {th }}$ crossing to its undercrossing arc; equivalently, it is the linking number of the two parts of the knot which the $j^{\text {th }}$ crossing, if split so as to give a link, separates.

- In the limit $q \longrightarrow 1$ with $q^{\lambda}$ (or $q^{\lambda+1}$ ) constant, we obtain a formal power series in $h=q-1$. In particular, the Kashaev invariant obtained by looking at the colored Jones polynomial in color $K$ at the $K^{\text {th }}$ root of unity $q=e^{\frac{2 \pi i}{K}}$ is obtained by setting $\mu=K, q^{\mu}=1$,

$$
K_{L}(q)=\sum_{\substack{l_{j}=0 \\
j=1, \ldots, c}}^{\infty} q^{c^{0}(\mathbf{l})} \prod_{j=1}^{c}\left(q^{-\sigma_{j}}-1\right) \ldots\left(q^{-\sigma_{j} l_{j}}-1\right)\left\{\begin{array}{c}
s(j)+l_{j} \\
l_{j}
\end{array}\right\}_{+}\left\{\begin{array}{c}
s(j)+l_{j} \\
l_{j}
\end{array}\right\}_{-}
$$

where $c^{0}(\mathbf{l})$ is the constant term in $c^{\mu}(\mathbf{l})=d(\mathbf{l}) \mu+c^{0}(\mathbf{l})$, namely

$$
c^{0}(\mathbf{l})=-d(\mathbf{l})-\sum_{\substack{a \\ k<i_{a}}} \delta_{a} \epsilon_{k} l_{k}-\frac{1}{2} \sum_{j} \sigma_{j} l_{j}-\frac{1}{2} \sum_{\substack{i \\ k<i}} \sigma_{i} \epsilon_{i} \epsilon_{k} l_{i} l_{k}+\sum_{\substack{j \\ i<j-, k<j+}} \sigma_{j} \epsilon_{i} \epsilon_{k} l_{i} l_{k}
$$

- When $\mu=2$, the colored Jones polynomial reduces to the one-variable Jones polynomial 10. It can be checked that the only non-zero contributions to the sum in Theorem 1 in this case come from $l_{j} \in\{0,1\}$ for which $-\sum_{k<i} \epsilon_{k} l_{k} \in\{0,1\}$ for all $i$. This sum is similar to (though different from) the beautifully simple state model given by Kauffman for the bracket polynomial in [11.

Put concisely, the colored Jones function depends on two parameters, $q$ and $\mu$, and one can make sense of it if at least one of the following holds: (i) $q$ is a root of unity; (ii) $\mu \in \mathbf{N}$; (iii) $q=1+h$ is considered as a formal parameter with expressions as formal power series in $h$ and $\mu$ is fixed; (iv) $q$ is considered as a formal parameter with expressions as formal power series in $h$ and $q^{\mu}$ is fixed. 


\section{The WRT and Ohtsuki invariants}

\subsection{The $S U(2)$ and $S O(3)$ 3-manifold invariants}

Suppose that $q$ is a $K^{\text {th }}$ root of unity. The prescription of [21] defines for a link $L$ with $c$ components and framing $f_{i}= \pm 1$ on the $i^{\text {th }}$ component,

$$
\langle L\rangle=\sum_{\mu_{1}, \ldots, \mu_{c}=1}^{K-1} J_{L_{0}}^{\prime}\left(\mu_{1}, \ldots, \mu_{c}\right) \prod_{i=1}^{c}\left[\mu_{i}\right]^{2} q^{\sum_{i} f_{i} \Omega\left(\mu_{i}\right)}
$$

where $q^{\Omega(\mu)}=f_{\mu}$ is the framing normalization, that is $\Omega(\mu)=\frac{\mu^{2}-1}{4}$.

The $S U(2)$ invariant of a 3-manifold $M$ obtained from $S^{3}$ by surgery around a link $L$ is then

$$
Z_{K}(M)=G_{+}^{-\sigma_{+}} G_{-}^{-\sigma_{-}}\langle L\rangle
$$

where $\sigma_{ \pm}$are the numbers of positive/negative eigenvalues of the linking matrix of $L$ and $G_{ \pm}$are the bracket values for the unknot with framings \pm 1 . When $L$ is a knot $(c=1)$ with framing $f= \pm 1$, so that $M$ is an integer homology 3 -sphere, this reduces to

$$
Z_{K}(M)=\frac{\sum_{\mu=1}^{K-1} q^{f \mu^{2} / 4}[\mu]^{2} J_{L_{0}}^{\prime}(\mu)}{\sum_{\mu=1}^{K-1} q^{f \mu^{2} / 4}[\mu]^{2}}
$$

The $S O(3)$ invariant is obtained by the same procedure except that the sums are all restricted to $\mu_{i}$ even; see [12].

\subsection{The $S U(2)$ perturbative invariant (Ohtsuki series)}

Following Le and Habiro, we now show how to obtain Habiro's cyclotomic form for the Ohtsuki series from Theorem 1. Assume that $M$ is obtained by $f= \pm 1$-surgery around a knot $L$, so that $M$ is an integer homology sphere.

The expression for $J_{L_{0}}^{\prime}(\mu)$ in Theorem 1 is a sum over $l$ of terms which are polynomial in $q^{\mu}$ and $q$ (and their inverses). A term $q^{m \mu}$ in $J_{L_{0}}^{\prime}(\mu)$ contributes

$$
\frac{\sum_{\mu=1}^{K-1} q^{f \mu^{2} / 4}[\mu]^{2} q^{m \mu}}{\sum_{\mu=1}^{K-1} q^{f \mu^{2} / 4}[\mu]^{2}}
$$


to $Z_{K}(M)$. Both summands vanish at $\mu=0$ and are even in $\mu$, so that the sum can be replaced by a complete period $2 K$. Completing the square, $\sum q^{f \mu^{2} / 4} q^{m \mu}=q^{-f m^{2}} \sum q^{f \mu^{2} / 4}$, thus the contribution to $Z_{K}(M)$ of a term $q^{m \mu}$ in $V_{L_{0}}^{\prime}(\mu)$ is

$$
\frac{q^{-f(m+1)^{2}}+q^{-f(m-1)^{2}}-2 q^{-f m^{2}}}{2 q^{-f}-2} \in \mathbf{Z}\left[q^{-f}\right] .
$$

Let $B$ be the ring of formal power series in $h=q-1$ whose coefficients are polynomials in $q^{ \pm \mu}$ with integer coefficients. For $f= \pm 1$, define a map $\phi_{f}: B \longrightarrow \mathbf{Z}[[h]]$ linear over $\mathbf{Z}[[h]]$ by

$$
\phi_{f}\left(q^{m \mu}\right)=\frac{q^{-f(m+1)^{2}}+q^{-f(m-1)^{2}}-2 q^{-f m^{2}}}{2 q^{-f}-2},
$$

so that $Z_{K}(M)=\phi_{f}\left(J_{L_{0}}^{\prime}(\mu)\right)$.

Lemma 1 For any $a, b \in \mathbf{Z}, l \in \mathbf{N}, \phi\left(q^{a \mu}\left(q^{\mu-b-1}-1\right) \ldots\left(q^{\mu-b-l}-1\right)\right)$ is divisible by the $l^{\text {th }}$ cyclotomic polynomial $\phi_{l}(q)$ in $\mathbf{Z}\left[q^{ \pm 1}\right]$.

Proof Suppose $\zeta$ is a root of unity of order $l$. It is sufficient to show that the expression in the lemma vanishes when evaluated at any such $q=\zeta$. Expanding out the product,

$$
\phi\left(q^{a \mu}\left(q^{\mu-b-1}-1\right) \ldots\left(q^{\mu-b-l}-1\right)\right)=\sum_{S \subset\{1, \ldots, l\}}(-1)^{l-|S|} q^{\Sigma S-b|S|} \phi\left(q^{(a+|S|) \mu}\right)
$$

where $\Sigma S$ and $|S|$ denote the sum of elements and the number of elements in the set $S$, respectively. Under the map $S \longmapsto S+1$, which adds 1 (modulo l) to every element of $S$, the number $\Sigma S$ increments by $|S|$ while $|S|$ stays unchanged. Under repeated application of this map, we get orbits of size $l$ (for $0<|S|<l$ ) and two orbits with one element, namely the empty set and the whole set. The contribution from any orbit of size $l$ contains a factor $\sum_{i=0}^{l-1} q^{i|S|}$ which vanishes when evaluated at $q=\zeta$. The contribution from the two size one orbits is

$$
(-1)^{l} \phi\left(q^{a \mu}\right)+q^{\frac{1}{2} l(l+1)-b l} \phi\left(q^{(a+l) \mu}\right) .
$$

When evaluated at $q=\zeta, \phi\left(q^{a \mu}\right)=\phi\left(q^{(a+l) \mu}\right)$ while $\zeta^{l}=1$ and $\zeta^{\frac{1}{2} l(l+1)}=$ $(-1)^{l+1}$, and again the contribution vanishes. 
Now for any $p \leq l, q^{a \mu}\left(q^{\mu-b-1}-1\right) \ldots\left(q^{\mu-b-l}-1\right)$ can be written as a linear combination (over $\mathbf{Z}\left[q^{ \pm 1}\right]$ ) of expressions of a similar form with chains of length $p, q^{a^{\prime} \mu}\left(q^{\mu-b^{\prime}-1}-1\right) \ldots\left(q^{\mu-b^{\prime}-p}-1\right)$. As a corollary, the expression in Lemma 1 is divisible by $\phi_{p}(q)$ for any $p \leq l$ and therefore also (since they are coprime) by $<l>$ ! $\equiv \prod_{p=1}^{l} \phi_{p}(q)$. Here $<l>! \in \mathbf{Z}[q]$ is the minimal monic polynomial in $q$ which vanishes at all roots of unity of order $\leq l$ (but not at $q=1$ ).

Corollary For any $a, b \in \mathbf{Z}, l \in \mathbf{N}, \phi\left(q^{a \mu}\left(q^{\mu-b-1}-1\right) \ldots\left(q^{\mu-b-l}-1\right)\right)$ is divisible by $<l>$ ! in $\mathbf{Z}\left[q^{ \pm 1}\right]$.

Lemma 2 For any integers $a, b_{j}, \phi_{f}\left(q^{a \mu} \prod_{j=1}^{l}\left(q^{\mu-b_{j}}-1\right)\right)$ is divisible by $h^{\left\lceil\frac{l}{2}\right\rceil}$.

Proof Let $\psi: B \longrightarrow \mathbf{Z}[[h]]$ be defined by $\psi\left(q^{m \mu}\right)=q^{m^{2}}$ for $m \in \mathbf{Z}$, extended linearly over $\mathbf{Z}[[h]]$. Write $q=e^{\hbar}$ and extend $\psi$ linearly to formal power series with rational coefficients. Then $\psi\left(e^{m \mu \hbar}\right)=e^{m^{2} \hbar}$. Comparing coefficients of powers of $m, \mu^{2 n} \longmapsto \frac{(2 n) !}{n ! \hbar^{n}}$ while $\mu^{2 n+1} \longmapsto 0$. In particular, for $s \leq r, \psi\left(\hbar^{r} \mu^{s}\right)$ is divisible by $h^{\lceil r / 2\rceil}$.

Now $\phi_{-1}$ and $\psi$ are related by

$$
\phi(f)=\frac{1}{2 h} \psi\left(\left(q^{\mu}+q^{-\mu}-2\right) f\right)=\frac{1}{2 h} \psi\left(\left(q^{\mu}-1\right)\left(q^{-\mu}-1\right) f\right)
$$

Expanding as a power series in $h$, whose coefficients are polynomials in $\mu$, $q^{a \mu} \prod_{j=1}^{l}\left(q^{\mu-b_{j}}-1\right)\left(q^{\mu}-1\right)\left(q^{-\mu}-1\right)$ is divisible by $h^{l+2}$, and such that the coefficient of $h^{r}(r \geq l+2)$ is a polynomial in $\mu$ of degree at most $r$. Replacing $h$ by $\hbar$ preserves these properties (though coefficients of powers of $\hbar$ may now be polynomials with non-integral, but rational, coefficients). Hence applying $\psi$ we obtain an element of $\mathbf{Q}[[\hbar]]$ divisible by $\hbar^{\left[\frac{l+2}{2}\right\rceil}$. The lemma now follows.

Lemma 3 For any integers $a, b_{j}, l_{j}$,

$$
\phi_{f}\left(q^{a \mu} \prod_{j}\left(q^{\mu-b_{j}-1}-1\right) \cdots\left(q^{\mu-b_{j}-l_{j}}-1\right)\right)
$$

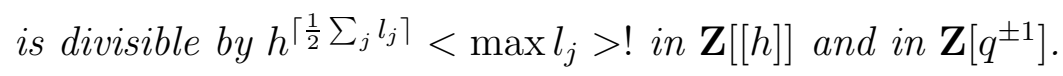


Proof Note that $\phi_{1}$ and $\phi_{-1}$ interchange when $q$ and $q^{-1}$ are interchanged, so it is sufficient to prove the lemma for $\phi \equiv \phi_{-1}$. Next observe that the expression in the lemma is actually a polynomial in $q^{ \pm 1}$ and so divisibility in $\mathbf{Z}[[h]]$ and in $\mathbf{Z}\left[q^{ \pm 1}\right]$ are equivalent. Since $h$ and $\langle n\rangle$ ! are coprime in $\mathbf{Z}[[h]]$, it is sufficient to prove divisibility by $h^{\left[\frac{1}{2} \sum_{j} l_{j}\right\rceil}$ and by $<\max l_{j}>$ ! separately; for the first we work in $\mathbf{Z}[[h]]$ (Lemma 2) and the second in $\mathbf{Z}\left[q^{ \pm 1}\right]$ (Corollary).

Putting Lemma 3 together with Theorem 1 from the last section gives the following.

Theorem 2 The WRT invariant of a manifold, $M$, obtained by $f= \pm 1$ surgery around a knot, $L$, is given by

$$
Z_{K}(M)=\sum_{\substack{l_{j}=0 \\
j=1, \ldots, c}}^{\infty} q^{c^{0}(\mathbf{l})} \phi_{f}\left(q^{d(\mathbf{l}) \mu} \prod_{j=1}^{c} \prod_{p=1}^{l_{j}}\left(q^{\sigma_{j}(\mu-s(j)-p)}-1\right)\right) \prod_{j=1}^{c}\left\{\begin{array}{c}
s(j)+l_{j} \\
l_{j}
\end{array}\right\}_{\sigma_{j}}
$$

in which the contribution from $\mathbf{l}$ is divisible by $h^{\left\lceil\frac{1}{2} \sum_{j} l_{j}\right\rceil}<\max l_{j}>$ !. Here $s(j)=-\sum_{i<j-} \epsilon_{i} l_{i}$ while $c^{0}(\mathbf{l})$ and $d(\mathbf{l})$ are given by (5) and (4) respectively. This is meaningful when $q$ is a root of unity (then the sum is finite as the only non-vanishing contributions are when $l_{j}<K$ for all $j$; the sum is the WRT invariant) and as a formal power series in $h$ (the sum is the Ohtsuki series, $\left.Z_{\infty}(M)\right)$.

In [7], Habiro obtains a more symmetric cyclotomic form for Ohtsuki series which is needed to prove integrality in general. For the case of surgery around knots, the above simple-minded approach is sufficient.

\subsection{Growth rates in Ohtsuki series}

Suppose that $M$ is an integral homology sphere obtained by surgery around a knot. Then the Ohtsuki series $Z_{\infty}(M)$ is a formal power series $\sum_{n=0}^{\infty} \lambda_{n}(M) h^{n}$ in $h=q-1$. In this section we use the formula given in Theorem 3 to determine a bound on the growth rate of coefficients $\lambda_{n}(M)$ with $n$. By the theorem, the only terms $\mathbf{l}$ which contribute to $\lambda_{N}(M)$ are those for which $\left\lceil\frac{1}{2} \sum_{j} l_{j}\right\rceil \leq N$, that is $\sum_{j} l_{j} \leq 2 N$; there are $\left(\begin{array}{c}c+2 N \\ c\end{array}\right)=O\left(N^{c}\right)$ such l's.

The double product in the summand in Theorem 2 is a product of $\sum_{j} l_{j}$ factors of the form $q^{ \pm \mu+a}-1$ where $a \in \mathbf{Z}$. Thus the contribution from $\mathbf{l}$ to 
$Z_{\infty}(M)$ can be expanded out to give a sum of $2^{\sum_{j} l_{j}} \leq 4^{N}$ terms of the form

$$
\pm \phi_{f}\left(q^{\alpha \mu+\beta}\right) \cdot \prod_{j}\left\{\begin{array}{c}
s(j)+l_{j} \\
l_{j}
\end{array}\right\}_{\sigma_{j}}
$$

where $\alpha=d(\mathbf{l})+\sum_{j} \sigma_{j}\left|P_{j}\right|$ and $\beta=c^{0}(\mathbf{l})-\sum_{j} \sigma_{j}\left(s(j)\left|P_{j}\right|+\Sigma\left(P_{j}\right)\right)$, while $P_{j} \subset\left\{1, \ldots, l_{j}\right\}$. Using (44) and (5), one finds estimates

$$
|\alpha| \leq 2 N\left(1+\max \left|q_{j}\right|\right)+\frac{b}{2},|\beta| \leq(16 c+10) N^{2}+2 N\left(2+\max \left|q_{j}\right|\right)+\frac{b}{2}
$$

that is, $\alpha=O(c N)$ and $\beta=O\left(c N^{2}\right)$, so that the order of the coefficient of $h^{n}(n \leq N)$ in $\phi_{f}\left(q^{\alpha \mu+\beta}\right)$ is that of $\left(\begin{array}{c}a c^{2} N^{2} \\ n+1\end{array}\right)$ ( $a$ is a constant).

The final term to estimate is of the coefficient of $h^{N-n}$ in the product of $c$ $q$-binomial coefficients on the right hand side of (6). Each of these $q$-binomial coefficients is of the form $\left\{\begin{array}{l}a \\ b\end{array}\right\}$ with $b \leq a \leq 2 N$; such a coefficient is a sum of $\left(\begin{array}{l}a \\ b\end{array}\right)$ powers of $q$ (with exponents at most $\left.b(a-b)\right)$ and so the coefficient of a power $h^{r}$ is at most

$$
\left(\begin{array}{c}
2 N \\
N
\end{array}\right)\left(\begin{array}{c}
N^{2} \\
r
\end{array}\right) \leq 4^{N}\left(\begin{array}{c}
N^{2} \\
r
\end{array}\right)
$$

Taking a product of $c$ such terms can accumulate in the coefficient of $h^{r}$ at most $4^{c N}\left(\begin{array}{c}c N^{2} \\ r\end{array}\right)$ and thus the coefficient of $h^{N}$ in (66) is at most

$$
\sum_{n=0}^{N}\left(\begin{array}{c}
a c^{2} N^{2} \\
n+1
\end{array}\right)\left(\begin{array}{c}
c N^{2} \\
N-n
\end{array}\right)=\left(\begin{array}{c}
\left(a c^{2}+c\right) N^{2} \\
N+1
\end{array}\right)
$$

Putting all the above estimates together gives the order of $\lambda_{N}(M)$ to be at most that of

$$
N^{c} \cdot 4^{N}\left(\begin{array}{c}
a c^{2} N^{2} \\
N+1
\end{array}\right)=x_{N}
$$

which is an order of growth of the bound for which $\frac{x_{N}}{x_{N-1}}$ asymptotically grows linearly with $N$, with slope of order $c^{2}$. 
Theorem 3 There exist bounds $x_{n}$ dependent only on $n$ and the number of crossings $c$ in the knot $K$, for which $\left|\lambda_{N}\left(S_{K}^{3}\right)\right|<x_{N}$ for all $N \in \mathbf{N}$ while $\lim _{n \rightarrow \infty} \frac{x_{N+1}}{N x_{N}}$ exists and is of order $c^{2}$.

This should be compared with the slope conjecture of [9] which states that $\frac{\lambda_{N}(M)}{\lambda_{N-1}(M)}$ is asymptotically linear in $N$, with coefficient, $\sigma(M)$, called the slope of $M$. Combining with the above result, it is reasonable to conjecture that $\sigma(M)$ is of order $c^{2}$, when $M$ is obtained by surgery around a knot with $c$ crossings. This is consistent with known results (9], 13]) on the slope for Seifert fibred manifolds.

\section{$5 \quad$ Examples}

In the literature (see 3], [5], 8], 15], 16]) there are computations of the colored Jones function for the simplest knots, the trefoil, figure- 8 knot and more generally, torus knots and twist knots. Applying Theorems 1 and 2, we reproduce the simplest of these results, although some are in different forms.

\subsection{The trefoil}

Suppose that $L$ is the right hand trefoil, presented as in Figure 6. In general, the formulae obtained will be simpler if the basepoint is chosen so that the first crossing encountered is an overcrossing (and the last before returning to the basepoint is an undercrossing. Crossing 3 is a left pointing crossing and the appropriate enhanced Gauss diagram is shown on the right in Figure 6.

In this case $c=3$ and the six points (from crossings) on the outer circle of the Gauss diagram are marked $1+, 2-, 3+, 1-, 2+$ and $3-$, respectively. Also there is just one blob $K^{-2}$, so that $b=1$ and $\delta_{1}=-1$. The signs of the crossings $\sigma_{j}$ are all +1 . Hence

$$
s(1)=l_{2}-l_{1}-l_{3}, \quad s(2)=-l_{1}, \quad s(3)=-l_{3} .
$$

Non-vanishing contributions to $V_{L_{0}}^{\prime}(\mu)$ appear only when $s(j)$ are all nonnegative, so that $l_{1}=l_{3}=0$. Then $s(1)=-l_{2}$ while $s(2)=s(3)=0$. We calculate $q_{2}=-2$ so that

$$
d(l)=-1-2 l, \quad c(l)=1+\frac{1}{2} l+\frac{1}{2} l^{2},
$$



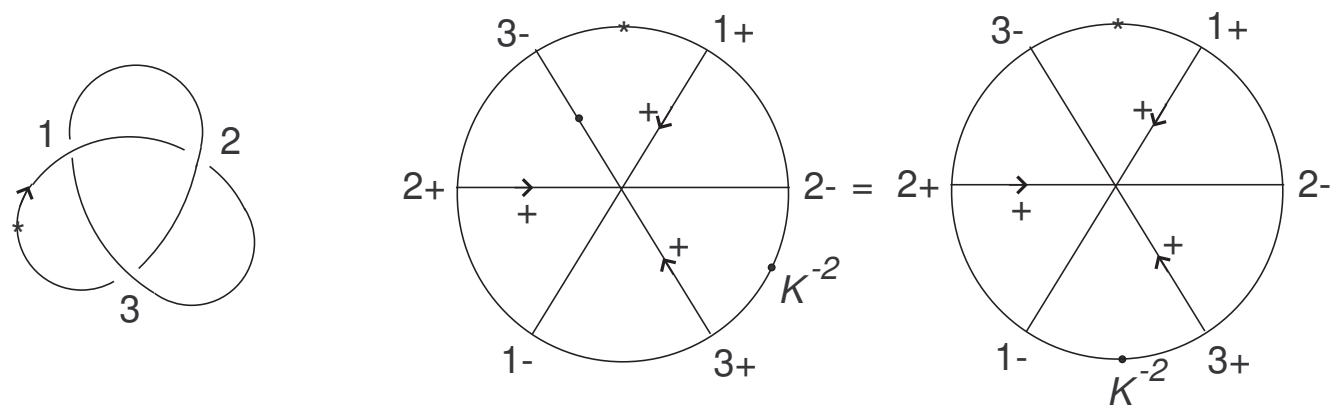

Figure 6: Trefoil knot and its enhanced Gauss diagram

and so $c^{\mu}(l)=-(1+2 l) \mu+\left(1+\frac{1}{2} l+\frac{1}{2} l^{2}\right)$. Theorem 1 now gives the Jones function of the trefoil as

$$
J_{L_{0}}^{\prime}(\mu)=\sum_{l=0}^{\infty} q^{-(2 l+1) \mu+\frac{l(l+1)}{2}+1}\left(q^{\mu-1}-1\right) \ldots\left(q^{\mu-l}-1\right),
$$

while the Kashaev invariant is

$$
K_{L}(q)=\sum_{l=0}^{\infty} q^{\frac{l(l+1)}{2}+1}\left(q^{-1}-1\right) \ldots\left(q^{-l}-1\right) .
$$

These formulae may be put in more standard form as

$$
J_{L_{0}}^{\prime}(\mu)=q \sum_{l=0}^{\infty} q^{-(l+1) \mu}\left(q^{1-\mu} ; q\right)_{l}, \quad K_{L}(q)=q \sum_{l=0}^{\infty}(1-q) \ldots\left(1-q^{l}\right),
$$

where $(a ; q)_{k}=(1-a)(1-a q) \ldots\left(1-a q^{k-1}\right)$. This formula was obtained by Habiro[7] and Le, as well as another one,

$$
J_{L_{0}}^{\prime}(\mu)=\sum_{k-0}^{\infty}(-1)^{k} q^{\frac{-k(k+3)}{2}} q^{-k \mu}\left(q^{\mu+1} ; q\right)_{k}\left(q^{\mu-1} ; q^{-1}\right)_{k}
$$

\subsection{The figure $8 \mathrm{knot}$}

The figure 8 knot $\left(4_{1}\right)$ is naturally presented in blackboard framing as a zero framed knot. Figure 5 shows its enhanced Gauss diagram along with the 
labeling of points on the circle. Here $c=4$ with signs $\sigma_{1}=\sigma_{3}=1$ and $\sigma_{2}=\sigma_{4}=-1$. There are two blobs $K^{-2}$ so that $b=2, \delta_{a}=-1$. Similar to the case of the trefoil, the only non-vanishing contributions come from $l_{1}=l_{3}=0$. Set $l_{2}=l$ and $l_{4}=m$. Then $s(1)=s(4)=l-m$ and $s(2)=s(3)=0$. Also $q_{1}=q_{3}=-1, q_{2}=q_{4}=1$ so that from (4)

$$
d(l, m)=l+m+1 .
$$

The last term in (5) requires an enumeration of all triples $(i, k, j)$ for which $i<j-$ and $k<j+$; the part of the list for which $i, k \in\{2 \pm, 4 \pm\}$ is $(2+, 2-, 3),(2+, 4+, 3),(2-, 2-, 3),(2-, 2-, 4),(2-, 4+, 3),(4+, 2-, 3)$, $(4+, 2-, 4),(4+, 4+, 3),(4-, 2-, 3)$ and $(4-, 4+, 3)$. Substituting into (5) gives

$c^{0}(l, m)=-(l+m+1)-l+(-l+m)+\frac{1}{2}(l+m)+\frac{1}{2}\left(-l^{2}-m^{2}\right)+\left(-l^{2}+l m\right)$

Theorem 1 supplies the Jones function of the knot $4_{1}$ as

$J_{L}^{\prime}(\mu)=\sum_{l \geq m \geq 0}^{\infty} q^{c^{\mu}(l, m)}\left(q^{1-\mu}-1\right) \ldots\left(q^{l-\mu}-1\right)\left\{\begin{array}{c}l \\ m\end{array}\right\}_{-}\left(q^{l-\mu-m+1}-1\right) \ldots\left(q^{l-\mu}-1\right)$

where $c^{\mu}(l, m)=(l+m+1) \mu-\frac{3}{2} l^{2}+l m-\frac{1}{2} m^{2}-\frac{5}{2} l+\frac{1}{2} m-1$. The Kashaev invariant is then

$$
K_{L}(q)=\sum_{l \geq m \geq 0} q^{c^{0}(l, m)}(q-1) \ldots\left(q^{l}-1\right)(q-1) \ldots\left(q^{m}-1\right)\left\{\begin{array}{c}
l \\
m
\end{array}\right\}_{+}\left\{\begin{array}{c}
l \\
m
\end{array}\right\}_{-}
$$

This is to be compared with Habiro [7] and Le's formulae

$$
J_{L}^{\prime}(\mu)=\sum_{k=0}^{\infty} q^{k \mu}\left(q^{-\mu-1} ; q^{-1}\right)_{k}\left(q^{-\mu+1} ; q\right)_{k}, \quad K_{L}(q)=\sum_{k=0}^{\infty}\left(q^{-1} ; q^{-1}\right)_{k}(q ; q)_{k}
$$

in which the symmetry $q \leftrightarrow q^{-1}$ due to the amphichirality of $4_{1}$ is apparent.

Acknowledgements The first author would like to thank Thang Le and Lev Rozansky for many conversations on the subject of computations of 3manifold invariants. The second author would like to acknowledge partial support from an Excellence Grant from the Hebrew University. 


\section{References}

[1] D. Bar-Natan, S. Garoufalidis, On the Melvin-Morton-Rozansky conjecture, Invent. Math. 125(1) (1996) 103-133.

[2] V. Drinfel'd, Quantum groups, Proceedings of the International Congress of Mathematicians (Berkeley, Calif., 1986), Amer. Math. Soc. (1987) 798-820.

[3] S. Garoufalidis, T. Le, The colored Jones function is q-holonomic, preprint arXiv:math.GT/0309214 (2003) to appear.

[4] S. Garoufalidis, M. Loebl, A non-commutative formula for the colored Jones function, preprint arXiv:math.QA/0411505 (2004) .

[5] K. Habiro, On the colored Jones polynomial of some simple links, Recent Progress Towards the Volume Conjecture, RIMS Kokyuroku 1172 (2000) .

[6] K. Habiro, Cyclotomic completions of polynomial rings, preprint arXiv:math.AC/0209324 (2002) .

[7] K. Habiro, On quantum $\mathrm{sl}_{2}$ invariants of knots and integral homology spheres, Geom. Topol. Monogr. 4 (2002) 55-68, arXiv:math.GT/0211044.

[8] K. Hikami, Difference equation of the colored Jones polynomial for torus knot, Int. J. Math. 15 (2004) 959-965, arXiv:math.GT/0403224.

[9] N. Jacoby, R. Lawrence, Some computations of Ohtsuki series, Proceedings of NATO Advanced Research Workshop "Advances in Topological Quantum Field Theory" (Kluwer, 2004) 53-70.

[10] V.F.R. Jones, Hecke algebra representation of braid groups and link polynomials, Annals Math. 126 (1987) 335-388.

[11] L.H. Kauffman, State models and the Jones polynomial, Topology 26 (1987) 395-407.

[12] R. Kirby, P. Melvin, The 3-manifold invariants of Witten and Reshetikhin-Turaev for sl(2, C, Invent. Math. 105(3) (1991) 473-545. 
[13] R. Lawrence, L. Rozansky, Witten-Reshetikhin-Turaev invariants of Seifert manifolds, Comm. Math. Phys. 205(2) (1999) 287-314.

[14] T. Le, Quantum invariants of 3-manifolds: integrality, splitting and perturbative expansion, Topology Appl. 127(1-2) (2003) 125-152, arXiv:math.QA/0004099.

[15] T. Le, The colored Jones polynomial and the A-polynomial of two-bridge knots, preprint arXiv:math.GT/0407521 (2004).

[16] G. Masbaum, Skein-theoretical derivation of some formulas of Habiro, Algebr. Geom. Topol. 3 (2003) 537-556, arXiv:math.GT/0306345.

[17] P.M. Melvin, H.R. Morton, The coloured Jones function, Comm. Math. Phys. 169(3) (1995) 501-520.

[18] H. Murakami, Quantum SU(2)-invariants dominate Casson's SU(2)invariant, Math. Proc. Camb. Phil. Soc. 115 (1993) 253-281.

[19] T. Ohtsuki, A polynomial invariant of integral homology 3-spheres, Math. Proc. Camb. Philos. Soc. 117(1) (1995) 83-112.

[20] N.Yu. Reshetikhin, V. Turaev, Ribbon graphs and their invariants derived from quantum groups, Comm. Math. Phys. 127 (1990) 1-26.

[21] N.Yu. Reshetikhin, V. Turaev, Invariants of 3-manifolds via link polynomials and quantum groups, Invent. Math. 103(3) (1991) 547-597.

[22] L. Rozansky, A contribution of the trivial connection to the Jones polynomial and Witten's invariant of $3 d$ manifolds I, II, Comm. Math. Phys. 175(2) (1996) 275-296, 297-318, arXiv: hep-th/9401061, arXiv:hep-th/9403021.

[23] L. Rozansky, Higher order terms in the Melvin-Morton expansion of the colored Jones polynomial, Comm. Math. Phys. 183(2) (1997) 291-306, arXiv:q-alg/9601009,

[24] E. Witten, Quantum field theory and the Jones polynomial, Commun. Math. Phys. 121 (1989) 351-399. 